\title{
Reply: Geospatial tools and Chagas control: A critical comment on the paper published under Weinberg et al., Evaluation and planning of Chagas control activities using geospatial tools. Geospat Health 2019;14:229-238
}

\author{
Diego Weinberg \\ Fundación Mundo Sano, Buenos Aires, Argentina
}

\begin{abstract}
We present here a reply on the observations and comments of Dr. David Gorla regarding our article "Evaluation and planning of Chagas control activities using geospatial tools" published in Geospatial Health 2019;14:229-238. (doi: 10.4081/gh.2019.786).
\end{abstract}

\section{Methodology used for collection of data used in the study}

The methodology for collection of data of infestation of triatomine bugs in a house using the Man/Hour $(\mathrm{M} / \mathrm{H})$ method is the most accepted method according to the literature and it is currently the most widely used by vector control programs in Latin America. This system for the detection of triatomine bugs is the one systematically used for large-scale interventions. In fact, the different indexes used to measure infestation are the ones that arise from the use of this method, and these same indexes are the ones managed by the community, scientific collaborations and public health effectors.

To state that this methodology is a source of false negatives is only to concentrate on one of the aspects of the mentioned method and goes against many published articles in the scientific literature that are a regular source of knowledge for the scientific community, equally useful for shaping health policies and public health effectors. With respect to the low sensitivity mentioned by Dr.

Correspondence: Diego Weinberg, Fundación Mundo Sano, Paraguay 1535 | C1061ABC, Buenos Aires, Argentina.

Conflict of interest: The Authors declare no potential conflict of interests.

Received for publication: 19 June 2020.

Accepted for publication: 19 June 2020.

(C) Copyright: the Author(s), 2020

Licensee PAGEPress, Italy

Geospatial Health 2020; 15:907

doi:10.4081/gh.2020.907

This article is distributed under the terms of the Creative Commons Attribution Noncommercial License (CC BY-NC 4.0) which permits any noncommercial use, distribution, and reproduction in any medium, provided the original author(s) and source are credited.
Gorla, citing a publication by Abad-Franch et al., 2014, it is important to highlight that in that study they did not use an irritant mixture to lure out the bugs. Precisely, in another study by the same author (Abad-Franch and Ferraz, 2010), they state that the use of irritants is an adequate additional tool to improve the sensitivity of the inspection method. In the published study in question, "Evaluation and planning of Chagas control activities using geospatial tools", the methodology for household inspections included the use of irritant mixtures.

Precisely the use of a method to determine infestation that corresponds to the standard protocol recommended by the Pan American Health Organization (PAHO), which is also systematically used in most of the vector control programs for Chagas, allows us to ponder on the strengths and weaknesses of the programs as they are currently designed.

\section{Experimental design for analysis and model used}

The experimental design used in our study is not categorized under a "real experiment"; on the contrary, the design could be classified as a quasi-experimental design or pre-experiment. It is valid to highlight that this design was not previously defined and it did not define the mechanism of data acquisition in the field since this study uses a 10-year database of field work from Mundo Sano Foundation. In this sense, the study provides value to the data obtained from the prevention and control programs previously designed and established.

We are aware of the limitations of quasi-experimental studies (i.e. history, maturation, testing), but their use is accepted by the scientific community and are even becoming more frequent (Harris et al., 2006). In fact, scientific literature on public health, social sciences and psychology, among others, provide many examples on ways to implement and improve quasi-experimental studies (Cook and Campbell, 1979; Trochim, 2001; Shadish et al., 2002).

Due to this design, the statistic association does not imply causality; we talk about an "apparent causal association". In particular, the design used in our study is of the type "one-group pretest/post-test design". According to this design, one case is observed in two moments, one before treatment and one after treatment. It is assumed that the change in the parameter under study is the result of the intervention, in this case treatment. Therefore, a control group was not used for comparison.

Although the known limitations of the study could be detailed more extensively with respect to its quasi-experimental design, we do state clearly that the analysis was performed using a dataset 
obtained in the framework of a steady program from Mundo Sano Foundation. If we take Dr. Gorla's proposal to the extreme, we would not be able to analyze data with any new tool if the data were not obtained using methodology specifically designed for that tool.

We believe that the existence of studies with pre-elaborated designs should be performed and are very important but that does not mean that studies like the one we published should not exist, using and taking advantage of pre-existent data, especially consistently obtained historical data such as the one used in this case.

With respect to Dr. Gorla's comments on the selection of the best model, we insist that this study did not pretend to analyze different models; instead we aimed to present a modelling strategy. In none of the sections of the study do we state that the model presented is the only one possible, we only suggest continuing to explore this path and these tools.

\section{Incorporating tools and geospatial analysis in the actions of surveillance and control programs}

In general, Dr. Gorla raises doubts or fears with respect to how this article might be perceived by the health system in order to really improve the efficacy of control programs. In this sense it is important to consider that this is a scientific publication and as such, it is one more grain added to the already existent literature on this matter. Therefore, it is important that the scientific community dedicated to this topic performs a comprehensive analysis of the knowledge obtained thus far. This should contribute to consensus and generation of practical recommendations that can shape and improve current guidelines and norms being used for the control of triatomine bugs with the aim of addressing the limitations and obtaining better results.

This study presents alternative ways to incorporate tools and geospatial analyses in the actions of vector surveillance and control. We also present alternative descriptive analyses for evaluation and planning of activities.

\section{Other comments}

With respect to factors not considered in this study (rural-urban migration, effect of the history of land-use change, effects of house quality/ house improvement), a subset of potentially influential factors related to the presence of triatomine bugs was actually performed, where the model to be used was defined. Some of the factors Dr. Gorla found missing have in fact been analyzed in a previous study (Weinberg et al., 2018). Additionally, our idea is to incorporate the analysis of complementary factors into a future study design.

Finally, with respect to Dr. Gorla's comment on the references, concretely "reports evaluating control programmes that consider the spatiotemporal history of infestation and spraying of a site are generally lacking in the literature" is not quite true. To name just a few articles that started appearing 15 years ago on spatiotemporal analysis of T. infestans infestations, i.e. Cecere et al. (2004), $\mathrm{Zu}$ Dohna et al. (2007, 2009), Levy et al. (2010), Provecho et al. (2017), Cavallo et al. (2018). We acknowledge that there is an error in the statement that will be corrected for clarity and interpretation. In this statement we refer to a "spatiotemporal history of BOTH infestation and spraying, and our vision and approach to the problem are related to the possibility of generating and discussing new analysis and tools that allow for continuous improvement of surveillance and control programs in Latin American countries, beyond the indexes, background and references.

We would like to thank Dr. Gorla for his comments, since, besides improving our article, he generated an instance to improve the clarity of the message that our study tried to contribute, not only to the health sector but also to the elaboration of public policy aimed at alleviating, in this case, the problems related to Chagas.

\section{References}

Abad-Franch F, Valenca-Barbosa, Sarquis O, Lima MM, 2014. All that glisters is not gold: sampling-process uncertainty in disease-vector surveys with false-negative and false-positive detections. PLoS Negl Trop Dis 8:e3187.

Abad-Franch F, Ferraz G, 2010. "Didn't you see that bug...?" Investigating disease vector occurrence when detection is imperfect. Revista Brasileira de Medicina Tropical 43 (Suppl II):31-4.

Harris AD, McGregor JC, Perencevich EN, FurunoJP, Zhu J, Peterson DE, Finkelstein J, 2006. The use and interpretation of quasi-experimental studies in medical informatics. J Am Med Inform Assoc 13:16-23.

Cook TD, Campbell DT, 1979. Quasi-experimentation: design and analysis issues for field settings. Houghton Mifflin.

Trochim WMK, 2001. Research methods knowledge base. Cincinnati: Atomic Dog Publishing. Available from: http://anatomyfacts.com/Research/ResearchMethodsKnowled geBase.pdf.

Shadish WR, Cook TD, Campbell DT, 2002. Experimental and quasi-experimental designs for generalized causal inference. Boston: Houghton Mifflin. Available from: https://moodle2.units.it/pluginfile.php/132646/mod_resource/ content/1/Estratto_ShadishCookCampbellExperimental2002.p df.

Weinberg D, Lanfri M, Scavuzzo CM, Abril M, Lanfri S, 2019. Evaluation and Planning of Chagas Control Activities Using Geospatial Tools. Geospat Health 14:229-38. doi: 10.4081/gh.2019.786.

Weinberg D, Porcasi X, Lanfri S, Abril M, Scavuzzo CM, 2018.Spatial analyzes of triatomine infestation indices and their association to the actions of a Chagas disease program and environmental variables during a 5-year intervention period. Acta Trop Dec 188:41-9. 